\title{
Algorithms for Sparse Network-based RTK GPS Positioning and Performance Assessment
}

\author{
Weiming Tang ${ }^{1}$, Xiaolin Meng ${ }^{2}$, Chuang Shi ${ }^{1}$ and Jingnan Liu ${ }^{1}$ \\ ${ }^{1}$ (GNSS Research Centre, Wuhan University, China) \\ ${ }^{2}$ (Nottingham Geospatial Institute, The University of Nottingham, UK) \\ (E-mail: Xiaolin.Meng@nottingham.ac.uk)
}

\begin{abstract}
The average inter-station distances in most established network Real Time Kinematic (RTK) systems are constrained to around $50 \mathrm{~km}$. A sparse network RTK system with an average inter-station distance of up to $300 \mathrm{~km}$ would have many appealing advantages over a conventional one, including a significant reduction in the development and maintenance costs. The first part of this paper introduces the key approaches for sparse network RTK positioning technology. These include long-range reference baseline ambiguity resolution and real-time kinematic ambiguity resolution for the rover receivers. The proposed method for long-range kinematic ambiguity resolution can overcome the network weaknesses through three procedures: application of the interpolated corrections from the sparse network only to wide-lane combination; searching the ambiguities of wide-lane combination; and searching L1 ambiguities with wide-lane combination and ionosphere-free observables. To test these techniques, a network including ten reference stations was created from the Ordnance Survey's Network (OS Net ${ }^{\mathrm{TM}}$ ) that covers the whole territory of the United Kingdom (UK). The average baseline length of this sparse network is about $300 \mathrm{~km}$. To assess the positioning performance, nine rover stations situated inside and outside the network were also selected from the OS Net ${ }^{\mathrm{TM}}$. Finally, the accuracy of interpolated corrections, the positioning accuracy and the initialization time required for precise positioning were estimated and analysed. From the observed performance of each rover receiver, and the accuracy of interpolated network corrections, it can be concluded that it is feasible to use a sparse reference station network with an average inter-station distance up to $300 \mathrm{~km}$ for achieving similar performance to traditional network RTK positioning. The proposed approach can provide more cost-efficient use of network RTK (NRTK) positioning for engineering and environmental applications that are currently being delivered by traditional network RTK positioning technology.
\end{abstract}

\section{KEY WORDS}
1. OS Net ${ }^{\mathrm{TM}}$
2. Sparse Network-based RTK.
3. Ambiguity Resolution
4. Network Corrections.

Submitted: 29 June 2012. Accepted: 19 September 2012.

1. INTRODUCTION. The use of network Real Time Kinematic (RTK) Global Positioning System (GPS) positioning has become an important solution for many high precision applications. For the best positioning services using current 
techniques, the average baseline length between reference stations has to be limited to between $30 \mathrm{~km}$ and $80 \mathrm{~km}$. However, setting up dense reference station networks in developing countries, or regions where there are access constraints, can pose difficulties. For instance, a selected reference station site must have a good observation environment, be stable, have access to power and have cabled or wireless internet or intranet. This means that it is normally very expensive to construct and manage a network of reference stations. At the same time, the demand for network RTK positioning services has been growing among the GPS user community which requires accurate and seamless kinematic position over longer distances. So it is necessary and useful to develop relevant techniques for sparse network RTK positioning and assess the resulting performance.

There are three essential technologies for achieving real-time positioning of centimetre accuracy using a sparse network. The first is the data processing for a long-range reference network to produce reliable RTK corrections, the second is the interpolation method of error models for the computation of the rover corrections according to the RTK corrections and finally the methods to be used for long-range real-time kinematic ambiguity resolution (AR) for the rover receivers.

Many researchers have proposed useful methods and developed relevant algorithms for long-range reference network ambiguity resolution. Sun et al. (1999) introduced a sequential least-square estimation algorithm for real-time network ambiguity resolution over a network of reference stations spaced $100 \mathrm{~km}$ to $200 \mathrm{~km}$ apart. Dai et al. (2003) and Chen et al. (2004) introduced the techniques for real-time integer ambiguity resolution using atmospheric delay derived from the system initialization process and the atmospheric biases estimated from previous epochs. $\mathrm{Hu}$ et al. (2005) proposed an ionosphere-weighted measurement model for instantaneous network ambiguity resolution with medium- to long-range receiver separations $(30 \mathrm{~km} \sim 130 \mathrm{~km})$.

Network-derived ionospheric and geometric corrections were employed in precise relative positioning (Cannon et al., 2001; Rizos, 2002; Wielgosz et al., 2004; Kashani I, 2004). Dai et al. (2004) summarized the available error interpolation methods for network RTK positioning. Kashani (2004) proposed that the double-differenced (DD) ionospheric delays estimated from the previous epoch with a successful integer fix can be used as the prediction for the current epoch, but a latency of 90 seconds seemed to be a limit for reliable instantaneous AR. Grgic et al. (2006) presented a method using a Low-Order Surface Model (LSC) that can significantly increase the interpolated correction accuracy. Mohino et al. (2007) developed a technique to estimate the vertical total electron content (VTEC) from the La Plata ionospheric model and so computed the slant total electron content (STEC) for receivers of unknown coordinates.

Long range RTK ambiguity resolution for rover receivers has been the important work in RTK GPS positioning. Kim et al. (2000) presented that there were 325 papers on ambiguity resolution and validation before 2000 for static, rapid static, kinematic and navigation applications. Edwards et al. (1999) presented a number of tests which, together, might form the basis of a methodology for assessing the performance of a RTK GPS system. Dai et al. (2000) proposed an integrated three-step procedure for the ambiguity resolution of medium-range baselines. Colombo et al. (2000) and Kashani et al. (2004) presented case studies for baselines over $100 \mathrm{~km}$. Dai et al. (2007) used partial search-and-fix strategies during the parameter estimation of a Kalman 
filter. Grejner-Brzezinska et al. (2005) developed a multiple reference station approach for regional differential kinematic GPS positioning. The primary processing algorithms used in this study include a weighted free-net (WFN) approach with the distance-dependent weighting scheme to derive optimal estimates of the user coordinates and realistic accuracy measures. The WFN approach is combined with a single epoch (instantaneous) ambiguity resolution algorithm for baselines exceeding $100 \mathrm{~km}$.

Previous research mainly focused on data processing and instantaneous ambiguity resolution of reference stations with inter-station distances of $100 \mathrm{~km}$ to $200 \mathrm{~km}$. For a sparse network RTK system with the inter-station distance up to $300 \mathrm{~km}$, novel methods and algorithms for network data processing and integer ambiguity resolution needed further study. This paper proposes a set of technologies that includes a Delaunay triangulation method to form the optimal network triangles, a three-step approach for double-differenced ambiguity resolution between reference stations and generation of network corrections, correction interpolation for the rover receivers and a long range kinematic ambiguity resolution algorithm. Using these techniques, a test network of ten reference stations and nine simulated rover stations was formed to assess the performance of proposed methodologies. The stations were all taken from Ordnance Survey's Network (OS Net ${ }^{\mathrm{TM}}$ ) in the UK; hence very accurate positions of all stations were readily available. Two datasets of one hour long were collected on 12 July 2008 and processed for presentation in this paper. The precision of interpolated ionospheric corrections for the rover sites, and the positioning precision and the initialization time are estimated and the affecting factors are also analysed.

2. SPARSE NRTK POSITIONING TECHNOLOGY. With respect to the standard network RTK, realization of the sparse network RTK requires several technical advances. These advances include carrier phase ambiguity resolution over long inter-station distances, bias interpolation method and user-end long distance kinematic ambiguity resolution method. The whole procedure for gathering the raw data from a network of reference stations and producing precise positioning results is a rather complicated process.

Figure 1 shows the overall processing procedure of sparse network RTK positioning technology.

In this paper, the following methods are applied to realize sparse network RTK positioning:

- The Delaunay triangulation method to form network cells (triangles);

- A three-step method for double-differenced (DD) ambiguity resolution of network RTK reference stations;

- A novel algorithm for the generation of high quality RTK corrections using a combined bias interpolation technique;

- A novel long distance kinematic ambiguity resolution method for the rover receivers.

Of these four strategies, the last one is the key. This method can overcome the inherent defects through three procedures: application of the interpolated corrections from the 


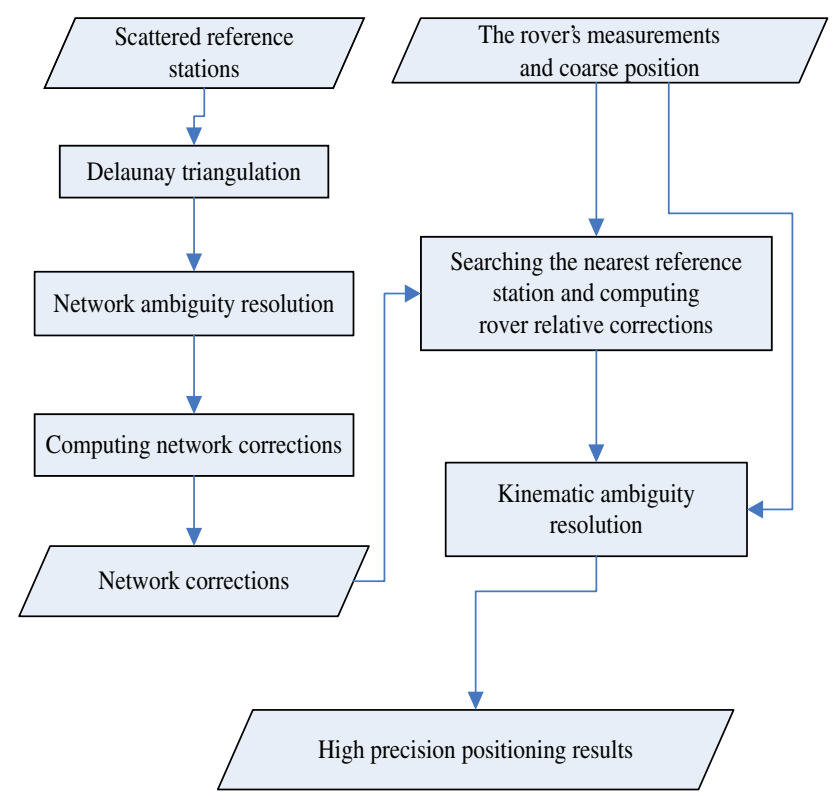

Figure 1. The procedure for sparse NRTK positioning.

sparse network only to wide-lane combination; searching the ambiguities of wide-lane combination; and searching L1 ambiguities with wide-lane combination and ionosphere-free observables.

2.1. The Delaunay Triangulation for the Formation of Network Triangles. The central processing software of a Network RTK system provides the network corrections according to the biases estimated from the measurements gathered from reference stations. It is necessary to form baselines from scattered reference stations for computing the DD biases. There are many algorithms to form baselines and triangles. However, in order to solve ambiguities easily and obtain reliable and highly accurate interpolation corrections, the criteria used in forming baselines is the shortest baseline length and the criteria for triangulation is the formation of an equilateral triangle. Delaunay triangulation can meet these requirements very well and the detailed procedure can be found from Cignoni (1998).

2.2. Long Distance Ambiguity Resolution Algorithm for Network Reference Stations. The resolution of ambiguities between reference stations includes widelane ambiguity resolution, initial narrow-lane ambiguity resolution and ambiguity resolution of L1 and L2 carrier phase measurements. This method has several attractive characteristics. For example, there is no need to solve observational equations, and ambiguity resolution is carried out on a satellite by satellite basis. The whole process of AR is as follows:

2.2.1. DD wide-lane ambiguity resolution. Firstly, the initial ambiguity of widelane combination is resolved using Melbourne-Wübbena combination (Hatch, 1982; Wübbena, 1985). The searching space is determined according to the relationship of Melbourne-Wübbena and pseudo-range ionosphere-free combinations. The integer wide-lane ambiguity is then validated by the accurate geometric range. 
2.2.2. Compute the initial ambiguity of DD narrow-lane combination and create the searching space. In the previous step, the ambiguity of wide-lane combination has been fixed to an integer. The coarse ionospheric delay of narrow-lane combination can be computed from the wide-lane combination and the accurate geometric range. Then the initial ambiguity of narrow-lane combination is computed. The searching space is formed according to the precision of the wide-lane combination and the characteristic that the ambiguities, both of wide- and narrow-lanes, are either odd or even numbers.

2.2.3. Ambiguity resolution of DD $L_{1}, L_{2}$ carrier phase measurements. After the searching space is created, the relationship between the two carrier phases is used as the criterion to validate the best ambiguity (Gao, 2002).

2.3. The method of computing systemic corrections for the rovers. The method adopted in this paper is called Modified Combined Bias Interpolation (MCBI) (Tang, 2006), which is based on the method of Combined Bias Interpolation (CBI) (Gao, 2002). The method of CBI does not separate tropospheric delay, ionospheric delay and other error sources. It cannot be applied to L1, L2 and widelane measurements simultaneously. MCBI separates the combined bias into three individual components: the first order ionospheric delay, the tropospheric delay estimated with the tropospheric model, and the residual error. The combined bias $\Delta \nabla R$ can be expressed as:

$$
\Delta \nabla R=\Delta \nabla d_{\text {ion }}+\Delta \nabla d_{\text {trop }}+\delta
$$

where $\Delta \nabla d_{\text {trop }}$ denotes the correction computed with the tropospheric model, $\Delta \nabla d_{\text {ion }}$ is the first order ionospheric delay, and $\delta$ is the sum of other residual errors including the higher order ionosphere delays, the residual error of the tropospheric model and the orbit error. By rearranging Eq. 1, the following equation can be obtained:

$$
\Delta \nabla R-\Delta \nabla d_{\text {trop }}=\Delta \nabla d_{\text {ion }}+\delta
$$

Let $\Delta \nabla R^{\prime}=\Delta \nabla R-\Delta \nabla d_{\text {trop }}$, then the new combined bias can be written as:

$$
\Delta \nabla R^{\prime}=\Delta \nabla d_{\text {ion }}+\delta
$$

The ionospheric delay and the residual errors can then be determined separately and applied to all measurements. The Linear Combination Model (LCM) (Dai et al., 2002) is applied to interpolate the ionospheric delay and the residual errors for the rover.

2.4. The Ambiguity Resolution Method for Long Range Baseline. This method consists of two parts: a two-step AR, where the first step is wide-lane AR and the second step is L1, L2 carrier phase AR; and, after eliminating the parameters of the coordinates, only the parameters of ambiguities are left. First the float ambiguities of wide-lane are computed using Melbourne-Wübbena combinations and then the integer ambiguities are searched out by the Least-squares AMBiguity De-correlation Adjustment (LAMBDA) method (Teunissen, et al., 1994, 1995a, 1995b). Secondly, wide-lane combinations are used to solve L1, L2 carrier phase ambiguities. In this process, in order to reduce the influence of ionospheric error, L1 ambiguities are expressed by the known wide-lane ambiguities and the ambiguities of ionosphere-free combinations. This method has two advantages: the first is that the ambiguities are integer and the second is that the influence of ionospheric error is largely reduced. 
2.4.1. Wide-lane ambiguities resolution. When the parameters of coordinates are eliminated, the equations of wide-lane ambiguities $\boldsymbol{Y}$ can be expressed as follows:

$$
\left[\begin{array}{c}
\boldsymbol{I} \cdot \lambda_{W L} \\
\tilde{\boldsymbol{B}}
\end{array}\right] Y=\left[\begin{array}{c}
\boldsymbol{L}_{M W} \\
\boldsymbol{L}_{W L}+\text { Cor }_{W L}
\end{array}\right]
$$

where $\tilde{\boldsymbol{B}}$ is the solution matrix, $\lambda_{W L}$ is the wavelength of wide-lane combination, $\boldsymbol{Y}$ is ambiguity vector, $\operatorname{Cor}_{W L}$ is referred to as the interpolated wide-lane combination correction, $\boldsymbol{I}$ is the unit matrix, $\boldsymbol{L}_{M W}$ is the Melbourne-Wübbena combination observation matrix, and $\boldsymbol{L}_{W L}$ is wide-lane observation matrix. The float ambiguities can be resolved with Equation 4. According to wide-lane float ambiguities and their covariance, multiple (usually 3 to 5) wide-lane integer ambiguities and their ratio values can be determined. Then the Sum of Squared Residuals (SOSR) can be computed and used to check the reliability of the best ambiguities. The criteria used to validate the wide-lane AR are:

$$
\left\{\begin{array}{l}
\text { Ratio }>M \\
V_{W L}^{T} P V_{W L}=\min
\end{array}\right.
$$

where $M$ is a definitive positive number and usually is $3 \cdot 0, V_{W L}$ is the remaining error vector.

2.4.2. L1, L2 ambiguities resolution. After fixing the wide-lane ambiguities, the ambiguity of ionosphere-free combination can be expressed by the ambiguity of L1 carrier phase and the ambiguity of wide-lane. The equations for resolving $\boldsymbol{L}_{1}$ ambiguities $\boldsymbol{Y}$ are:

$$
\left[\begin{array}{c}
\boldsymbol{I} \cdot \lambda_{L 1} \\
\tilde{\boldsymbol{B}}
\end{array}\right] \boldsymbol{Y}=\left[\begin{array}{c}
\boldsymbol{L}_{W L}+\operatorname{Cor}_{W L} \\
\boldsymbol{L}_{L C}
\end{array}\right]
$$

where $\tilde{\boldsymbol{B}}$ is the solution matrix, $\lambda_{L 1}$ is the wavelength of $\boldsymbol{L}_{1}, \boldsymbol{Y}$ is the ambiguity vector, $\boldsymbol{I}$ is the unit matrix, $\boldsymbol{L}_{W L}$ is the virtual observation vector which is computed by widelane combinations, and $\boldsymbol{L}_{L C}$ is the ionosphere-free observables.

The float ambiguities of $\boldsymbol{L}_{1}$ and the covariance are obtained through solving Eq. 6. Multiple (usually 3 to 5) integer ambiguities and their Ratios can be determined by the LAMBDA method. Then the SOSR can be used to check the successful rate of ambiguity resolution. For a long range baseline, in order to reduce the influence of ionosphere delay, the SOSR of ionosphere-free can be computed and compared. The criteria used to validate the ionosphere-free AR are:

$$
\left\{\begin{array}{l}
\text { Ratio }>M \\
V_{L C}^{T} P V_{L C}=\min \\
\left(k N_{2}+b\right)-N_{1}<\delta
\end{array}\right.
$$

where $M$ is a definitive positive number and usually is $3 \cdot 0, V_{L C}$ is the remaining error vector, $\delta$ is a definitive positive number, $\left(k N_{2}+b\right)-N_{1}$ is the difference of $N_{2}$ and $N_{1}, k$ and $b$ are constant values $(\mathrm{Gao}, 2002)$ which are computed by the relationship of $N_{2}$ and $N_{1}$ with the known station coordinates.

\section{EXPERIMENTAL DATA AND RESULT ANALYSIS}

3.1. OS Net ${ }^{\mathrm{TM}}$ of the UK. The Ordnance Survey of the UK operates a network of more than 150 continuously operating GPS receivers - OS Net ${ }^{\mathrm{TM}}$ as shown in Figure 2. 


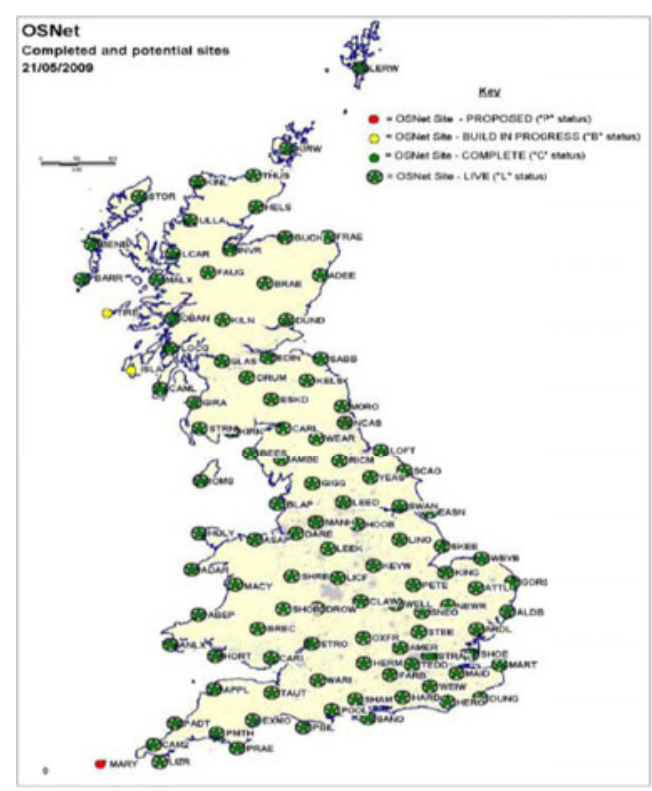

Figure 2. Ordnance Survey Active GPS Network (http://www.bigf.ac.uk/).

The Ordnance Survey is working with its partner organizations to utilise the GPS data from this network for the development and provision of a basket of real-time and post-process GPS correction services, with a choice of accuracy levels available to customers in a wide variety of application markets.

3.2. Use of OS Net ${ }^{\mathrm{TM}}$ to form a Sparse Network. In order to assess the performance of a sparse network RTK system covering the United Kingdom, ten reference stations were selected from the reference stations of OS Net ${ }^{\mathrm{TM}}$. The average interstation distance was around $300 \mathrm{~km}$. One Hz GPS measurements collected from these stations on July 12, 2008 were used in the test. The data were collected in two sessions: 00:00-01:00 UT (1-2 am local time), when the TEC is the lowest, and 14:00-15:00 UT (3-4 pm local time), when the TEC is at its highest in the day. Another nine stations were also selected from the OS $\mathrm{Net}^{\mathrm{TM}}$ as the rovers. Of these nine stations, six sites were located within the network and three were outside. The rover stations did not contribute to the estimation of the atmospheric corrections. The map of the reference network and the locations of the stations simulating rovers are presented in Figure 3. The receivers used at all reference stations were LEICA SR530 units. The sampling rate of the data was $1 \mathrm{~Hz}$. The antenna of station LERW was ASH700936D_M and the antenna of station OSHQ was ASH700936E. The antennae of the remaining stations were LEIAT504.

The analysis was performed in a post-processing mode; however, the algorithm is suitable for real-time application. Naturally, for a real RTK implementation, the issue of communication links and the computing power at the rover stations must be addressed properly.

The strategy used in the sparse network data processing is the same as that introduced in Figure 1. The triangle network is formed by the Delaunay Triangulation method discussed earlier. There are a total of 17 baselines in the network. The average baseline length is $300 \mathrm{~km}$. The longest length is $378 \cdot 3 \mathrm{~km}$ and the shortest is $226 \cdot 6 \mathrm{~km}$. 


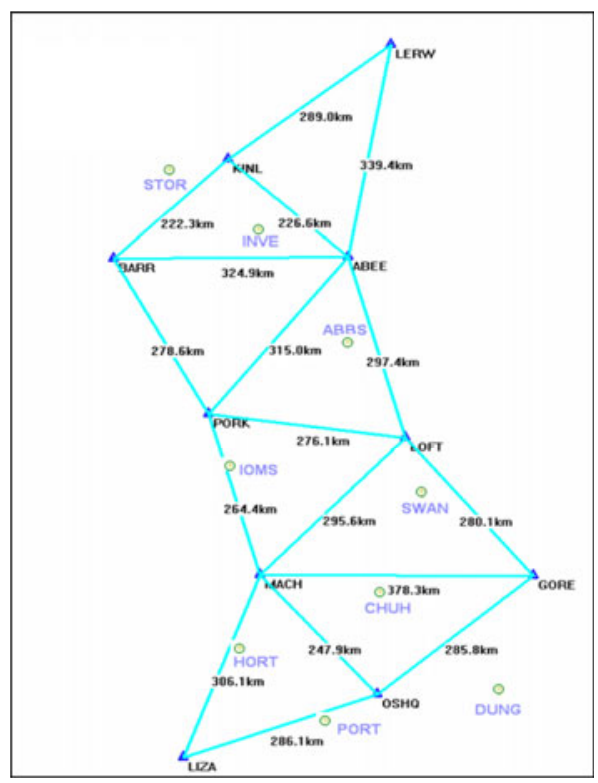

Figure 3. A Sparse Testing Network of the UK.

The service area covers England and Scotland. These 17 baselines were solved separately using the long-range ambiguity resolution method introduced earlier. All the ambiguities in a triangle were checked for whether the sum of a satellite's ambiguities is zero. If it was not zero, the ambiguities would be reprocessed. The corrections between reference stations were computed and stored into a file.

The algorithm used for the data processing of the rover measurements consists of several steps. The first step is that the rover's coarse position is computed in a single positioning mode. According to this position, the nearest reference station is selected as the master station and the relative corrections between the rover and the master station are interpolated.

3.3. The AR Performance of the Network. The average of times for first fix (Over 6 or all satellites are available) is $7 \mathrm{~s}$. None of the ambiguities fixed is wrong by comparing with the post-processing results of software Bernese 5.0. The values of most DD ionospheric biases are between than $+0.4 \mathrm{~m}$ and $-0.4 \mathrm{~m}$. The values of most DD tropospheric errors are less than $0.05 \mathrm{~m}$. Generally, the satellites with the lower elevation angles have bigger DD ionospheric biases and DD tropospheric errors.

3.4. The Precision of DD Ionosphere Delay. The precision of DD ionosphere corrections affects kinematic ambiguity resolution on the rover receivers and the performance of sparse network RTK positioning. In order to analyse the precision of interpolated ionosphere corrections, we firstly computed the corrections for the nine rovers with the known coordinates. Because the rovers are also reference stations, the coordinates of these rovers are precisely known. Then, the interpolated ionosphere corrections were computed using the methods introduced in the previous sections. The difference between the known corrections and the interpolated ones can be computed on an epoch-by-epoch and satellite-by-satellite basis. The average difference of each data session is given in Table 1 and Figure 4. In Table 1 the column entitled "In" denotes whether the rover is inside the network $(Y)$ or outside $(\mathrm{N})$. 
Table 1. The precision of interpolated ionosphere corrections.

\begin{tabular}{|c|c|c|c|c|c|}
\hline \multirow[b]{2}{*}{ Station Name } & \multicolumn{2}{|c|}{ Difference $(\mathrm{cm})$} & \multirow[b]{2}{*}{ In } & \multicolumn{2}{|c|}{ Nearest Ref. Station } \\
\hline & Sess. 1 & Sess. 2 & & Name & Dist.(Km) \\
\hline STOR & $2 \cdot 6$ & $3 \cdot 0$ & $\mathrm{~N}$ & KINL & $84 \cdot 5$ \\
\hline INVE & $2 \cdot 2$ & $2 \cdot 8$ & $\mathrm{Y}$ & KINL & $118 \cdot 2$ \\
\hline ABBS & $3 \cdot 2$ & $2 \cdot 4$ & $\mathrm{Y}$ & ABEE & $138 \cdot 3$ \\
\hline IOMS & $2 \cdot 7$ & $2 \cdot 2$ & $\mathrm{Y}$ & PORK & $91 \cdot 3$ \\
\hline SWAN & $3 \cdot 1$ & $2 \cdot 3$ & $\mathrm{Y}$ & LOFT & $96 \cdot 1$ \\
\hline $\mathrm{CHUH}$ & $3 \cdot 4$ & $3 \cdot 2$ & $\mathrm{Y}$ & OSHQ & $157 \cdot 4$ \\
\hline HORT & $3 \cdot 4$ & $2 \cdot 4$ & $\mathrm{Y}$ & MACH & $120 \cdot 5$ \\
\hline PORT & $3 \cdot 5$ & $2 \cdot 0$ & $\mathrm{~N}$ & OSHQ & $86 \cdot 1$ \\
\hline DUNG & $5 \cdot 3$ & $3 \cdot 3$ & $\mathrm{~N}$ & OSHQ & $170 \cdot 6$ \\
\hline
\end{tabular}

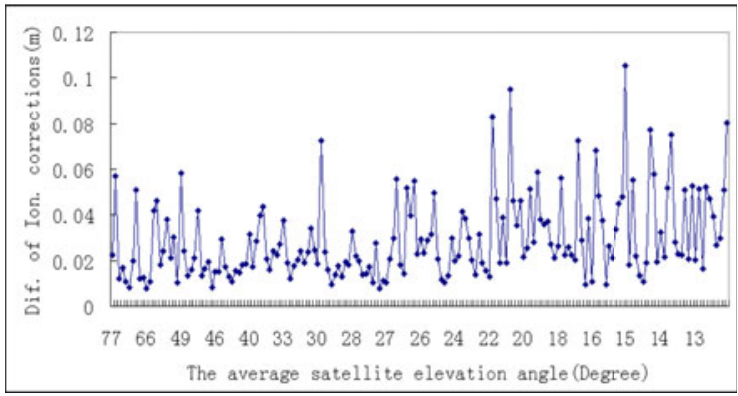

Figure 4. The one session average differences of all satellites.

In Table 1 the average precision of these interpolated corrections is about $3 \cdot 0 \mathrm{~cm}$. The precision of the rovers closer to a reference station or inside the network is more stable. The worst precision is $5 \cdot 3 \mathrm{~cm}$ attained from rover DUNG. DUNG is outside the network and the distance to the nearest reference station is $170.6 \mathrm{~km}$. The average precision of interpolated corrections of the six rovers inside the network is $2.8 \mathrm{~cm}$.

In order to analyse the relationship between satellite elevation angle and interpolation precision, the average correction difference of each satellite is computed and shown in Figure 4.

In Figure 4, the correction difference is increasing with the lower satellite elevation angle. When the satellite elevation angle is less than 20 degrees, half of the differences are more than $5 \mathrm{~cm}$.

3.5. Simulated Real-time Positioning Results. In order to test the performance of positioning, the positioning fixes of the nine rovers have been compared with their known coordinates. The initialisation time and the Three-Dimensional (3D) precision are listed at Table 3. Session 1 in this table refers to the data collected during 00:00 01:00 UT and Session 2 the data set between 14:00 15:00 UT. The rover locations are shown in Figure 2.

From Table 2, the average initialisation time is about $12.4 \mathrm{~s}$ and the maximum initialization time is $35 \mathrm{~s}$. The average precision of all rovers is $1.4 \mathrm{~cm}$ in the north direction, $1.3 \mathrm{~cm}$ in the east direction and $3.5 \mathrm{~cm}$ in the vertical direction respectively. The maximum error of all rovers in the vertical direction is $6.5 \mathrm{~cm}$ at rover INVE 
Table 2. Statistics of the rover positioning results.

\begin{tabular}{|c|c|c|c|c|c|}
\hline \multirow[b]{2}{*}{ Station Name } & \multirow[b]{2}{*}{ Session } & \multirow[b]{2}{*}{ Initial Time } & \multicolumn{3}{|c|}{ Accuracy of Positioning (cm) } \\
\hline & & & $\mathrm{N}$ & E & $\mathrm{U}$ \\
\hline \multirow[t]{2}{*}{$\mathrm{CHUH}$} & 1 & $8 \mathrm{~s}$ & $1 \cdot 5$ & $1 \cdot 2$ & $4 \cdot 4$ \\
\hline & 2 & $13 \mathrm{~s}$ & $0 \cdot 8$ & $0 \cdot 8$ & $2 \cdot 4$ \\
\hline \multirow[t]{2}{*}{ INVE } & 1 & $4 \mathrm{~s}$ & $1 \cdot 2$ & $1 \cdot 7$ & $6 \cdot 5$ \\
\hline & 2 & $4 \mathrm{~s}$ & $1 \cdot 0$ & $0 \cdot 6$ & $3 \cdot 0$ \\
\hline \multirow[t]{2}{*}{ STOR } & 1 & $9 \mathrm{~s}$ & $1 \cdot 3$ & 1.5 & $5 \cdot 5$ \\
\hline & 2 & $12 \mathrm{~s}$ & $0 \cdot 6$ & $0 \cdot 7$ & $2 \cdot 8$ \\
\hline \multirow[t]{2}{*}{ ABBS } & 1 & $6 \mathrm{~s}$ & $1 \cdot 3$ & $0 \cdot 8$ & $5 \cdot 8$ \\
\hline & 2 & $11 \mathrm{~s}$ & $1 \cdot 1$ & 1.5 & $2 \cdot 3$ \\
\hline \multirow[t]{2}{*}{ SWAN } & 1 & $5 \mathrm{~s}$ & $1 \cdot 1$ & $1 \cdot 3$ & $2 \cdot 7$ \\
\hline & 2 & $8 \mathrm{~s}$ & $0 \cdot 8$ & $1 \cdot 0$ & $4 \cdot 6$ \\
\hline \multirow[t]{2}{*}{ DUNG } & 1 & $18 \mathrm{~s}$ & $1 \cdot 7$ & $1 \cdot 2$ & $4 \cdot 3$ \\
\hline & 2 & $15 \mathrm{~s}$ & $2 \cdot 2$ & $3 \cdot 6$ & $4 \cdot 0$ \\
\hline \multirow[t]{2}{*}{ PORT } & 1 & $35 \mathrm{~s}$ & $2 \cdot 5$ & 1.5 & $4 \cdot 0$ \\
\hline & 2 & $6 \mathrm{~s}$ & $1 \cdot 1$ & $0 \cdot 7$ & $1 \cdot 8$ \\
\hline \multirow[t]{2}{*}{ HORT } & 1 & $23 \mathrm{~s}$ & $2 \cdot 5$ & $1 \cdot 5$ & $2 \cdot 3$ \\
\hline & 2 & $19 \mathrm{~s}$ & $1 \cdot 8$ & $1 \cdot 3$ & $2 \cdot 4$ \\
\hline \multirow[t]{2}{*}{ IOMS } & 1 & $14 \mathrm{~s}$ & $1 \cdot 1$ & $0 \cdot 6$ & $3 \cdot 3$ \\
\hline & 2 & $13 \mathrm{~s}$ & $1 \cdot 2$ & $1 \cdot 3$ & $1 \cdot 1$ \\
\hline \multicolumn{2}{|l|}{ Average } & $12 \cdot 39 \mathrm{~s}$ & $1 \cdot 37$ & $1 \cdot 26$ & $3 \cdot 50$ \\
\hline
\end{tabular}

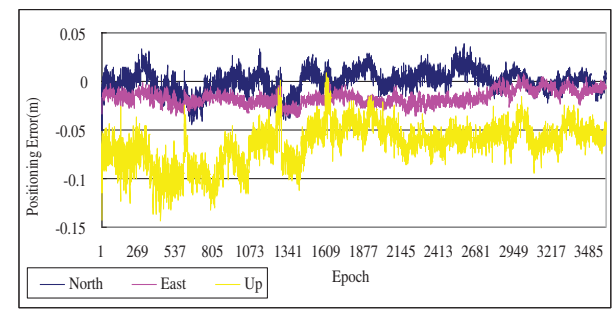

Figure 5. 3D positioning errors of rover INVE.

during Session 1. The second largest vertical error estimated from rover STOR in Session 1 is $5.54 \mathrm{~cm}$. The time series of 3 Dimensional (3D) errors of rover INVE are shown in Figure 5 and those of STOR in Figure 6.

In Figure 5 and Figure 6, there are obvious systemic offsets in the time series of height component. If these offsets are removed $(-0.064 \mathrm{~m}$ in Figure 6 and $-0.053 \mathrm{~m}$ in Figure 7), the positioning RMS of INVE is $0.017 \mathrm{~m}$ and that of STOR is $0.010 \mathrm{~m}$. The main cause for this is the effect of residual tropospheric error since the distance between the rover and reference stations is over $100 \mathrm{~km}$ and the model used cannot effectively remove tropospheric delay. The average of all the rovers' positioning RMS from both sessions was plotted against the distances to the nearest reference stations in Fig. 7. 


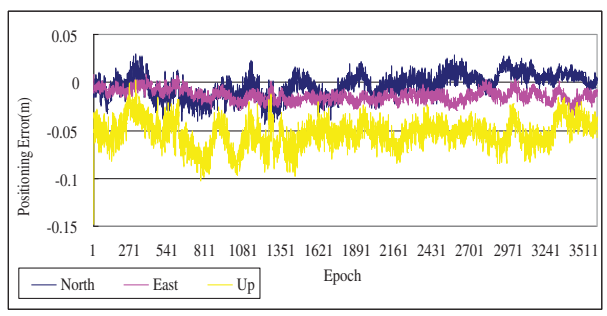

Figure 6. 3D positioning errors of rover STOR.

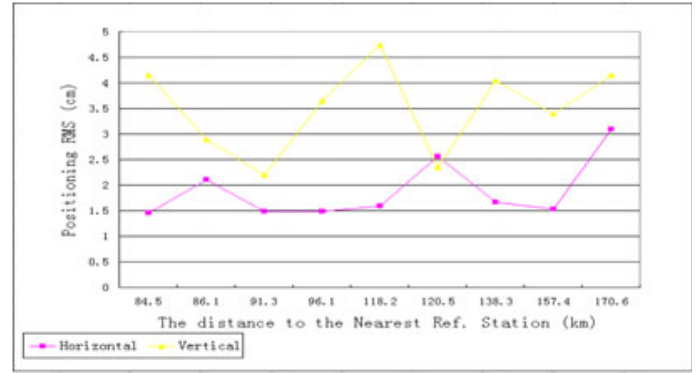

Figure 7. Positioning RMS against distances to the nearest reference stations.

In Figure 7, however, we have not taken account of the work by Zheng et al. (2005) which has shown a certain correlation between base-rover distances and tropospheric interpolation errors based on the analysis of a network of 129 stations. It is possible that the sample number is statistically too small to reliably demonstrate correlation.

4. CONCLUSIONS. A complete set of positioning technologies for sparse network-based RTK is proposed in this paper. To test these methods, a sparse network consisting of ten reference stations was created from OSNet ${ }^{\mathrm{TM}}$. The precision of both interpolated corrections and positioning results were calculated. From these results and analysis, the following conclusions can be drawn:

- The precision of interpolated corrections in this sparse network is about $3 \mathrm{~cm}$ and has a high correlation with the satellite elevation angle. The horizontal positioning precision is $1 \mathrm{~cm}$ to $2 \mathrm{~cm}$ and the vertical $3 \mathrm{~cm}$ to $5 \mathrm{~cm}$. This precision is equivalent to that of a traditional network RTK system. So, it is feasible to establish a sparse network RTK system with inter-station distances up to $300 \mathrm{~km}$ to cover the whole territory of the UK.

- In this sparse network, the precision of interpolated corrections for satellites with low elevation angles is poor, and the precision for some satellites exceeds $10 \mathrm{~cm}$ as shown in Figure 4. To overcome this problem, a novel method is proposed. Firstly, the interpolated corrections are used to correct wide-lane combinations and the error of $10 \mathrm{~cm}$ does not influence the wide-lane ambiguity resolution. After fixing wide-lane ambiguities, both wide-lane and ionosphere free observations are used in the L1 ambiguity resolution. Finally, the positioning 
results are computed using ionosphere free observations. The results of the testing network have proved the method is practical and efficient.

- The algorithms proposed are suitable for network-based real-time applications. Although the data used were previously recorded, all the computations were carried out in a simulated real-time processing mode.

- The precision of some interpolated corrections is close to $10 \mathrm{~cm}$ and some positioning results in the vertical direction have a systemic offset. How to further improve the precision of the interpolated corrections and eliminate or estimate these systemic errors will be addressed in future work.

- Although the systems of this paper are designed to perform adequately under "worst-case" conditions, the test data was from the OSNet ${ }^{\mathrm{TM}}$ of July 12, 2008 and the year 2008 was a relatively quiet solar year. The authors will test the method to perform under severe ionosphere storm conditions in their future work.

\section{ACKNOWLEDGEMENTS}

Ordnance Survey of the UK is acknowledged for providing the testing data sets of OSNet ${ }^{\mathrm{TM}}$. This research is funded by National Natural Science Foundation of China (No: 41004014), the national ' 863 High Technology Project' of China (No. 2012AA12A202) and Supported by the 111 Project (No: B07037). Air Commodore Norman Bonnor is acknowledged for proofreading this article.

\section{REFERENCES}

Cannon, M. E., Lachapelle, G., Fortes, L. P., Alves, P. and Townsend, B. (2001). The Use of Multiple Reference Station VRS for Precise Kinematic Positioning. Proceedings of the Japan Institute of Navigation, GPS Symposium 2001, 14-16 November, Tokyo, Japan, 29-37.

Chen, H., Rizos, C. and Han, S. (2004). An Instantaneous ambiguity resolution procedure suitable for medium-scale GPS reference station networks. Survey Review, 37(291), 396-410.

Cignoni, P., Montani, C. and Scopigno, R. (1998). DeWall: A fast divide and conquer Delaunay triangulation algorithm in ED. Computer-Aided Design, 30(5), 333-341. doi:10.1016/S0010-4485(97) 00082-1.

Colombo, O. L., Hernandez-Pajares, M., Juan, J. M. and Sanz, J. (2000). Ionospheric Tomography Helps Resolve GPS Ambiguities on the Fly At distances Of Hundreds of Kilometers During Increased Geomagnetic Activity. Proceedings of the IEEE PLANS 2000 Symposium, San Diego, California.

Dai, L. (2000). Dual-Frequency GPS/GLONASS Real-Time Ambiguity Resolution for Medium-Range Kinematic Positioning. Proceedings of the Institute of Navigation GPS 2000, 19-22 September, Salt Lake City, Utah.

Dai, L., Wang, J., Rizos, C. and Han, S. (2003). Predicting atmospheric biases for real-time ambiguity resolution in GPS/GLONASS reference station networks. Journal of Geodesy, 76(11-12), 617-628. doi: 10.1007/s00190-002-0286-1.

Dai, L., Han, S., Wang, J. and Rizos, C. (2004). Comparison of Interpolation Algorithms in NetworkBased GPS Techniques. Journal of the Institute of Navigation, 50(4), 277-293.

Dai, L., Eslinger, D. and Sharpe, T. (2007). Innovative Algorithms to Improve Long Range RTK Reliability and Availability. Journal of the Institute of Navigation NTM2007, 22-24 January, San Diego, California, 860-872.

Dong, D. N. and Bock, Y. (1989). Global Positioning System network analysis with phase ambiguity resolution applied to crustal deformation studies in California. Journal of Geophysical Research, 94(B4), 3949-3966. doi:10.1029/JB094iB04p03949.

Edwards, S. J., Cross, P. A., Barnes, J. B. and Bétaille. A Methodology for Benchmarking Real Time Kinematic GPS. Survey Review, 35, 163-174. 
Gakstatter, E. (2009). Technology to watch. GPS World, 20(5), 33-33.

Gao, X., Liu, J. and Ge, M. (2002). An Ambiguity Searching Method Using Singe Epoch for Network RTK Reference Stations. Acta Geodetica et Cartographica Sinica, 31(4), 305-309.

Goad, C. C. (1992). Robust techniques for determining GPS phase ambiguities. Proceedings of the 6th International Geodetic Symposium on Satellite Positioning, Columbus, Ohio, 17-20 March, 245-254.

Grejner-Brzezinska, D. A., Kashani, I. and Wielgosz, P. (2004). Analysis of the Network Geometry and Station Separation for Network-Based RTK. Journal of the Institute of Navigation NTM2004, January 26-28, San Diego, California, 469-474.

Grejner-Brzezinska, D. A., Kashani, I. and Wielgosz, P. (2005). On accuracy and reliability of instantaneous network RTK as a function of network geometry, station separation, and data processing strategy. GPS Solutions, 9, 212-225. doi: 10.1007/s10291-005-0130-1.

Grgic, P. M., Kealy, A., Gordini, C., Hale, M. and Retscher, G. (2006). Atmospheric Bias Interpolation for Network RTK GPS. IGNSS Symposium 2006, 17-21 July 2006, Holiday Inn, Surfers Paradise, Queensland, Australia.

Han, S. (1997). Carrier Phase-Based Long-Range GPS Kinematic Positioning. Dissertation, School of Geomatic Engineering, the University of New South Wales.

Hatch, R. (1982). Synergism of GPS code and carrier measurements. Proceedings of the Third International Geodetic Symposium on Satellite Doppler Positioning, New Mexico State University, 1213-1232.

Hatch, R. (1986). Dynamic differential GPS at the centimetre level. Proceedings of the 4th International Geodetic Symposium on Satellite Positioning, 28 April-2 May 1986, Las Cruces, New Mexico, 12871298.

Hu, G. and Abbey, D. A. (2005). An approach for instantaneous ambiguity resolution for medium- to longrange multiple reference station networks. GPS Solutions, 9(1), 1-11. doi:10.1007/s10291-004-0120-8.

Kim, D. and Langley, R. B. (2000). GPS Ambiguity Resolution and Validation: Methodologies, Trends and Issues. Proceedings of the 7th GNSS Workshop-International Symposium on GPS/GNSS, 30 November - 2 December 2000, Tutorial/Domestic Session, Seoul, Korea, 213-221.

Kashani, I. and Wielgosz, P. (2004). Towards Instantaneous Network-Based RTK GPS Over $100 \mathrm{~km}$ Distance. Proceedings of the Institute of Navigation 60th Annual Meeting, 7-9 June 2004, Dayton, Ohio.

Li, Z. and Gao, Y. (1998). Improving ambiguity resolution for a regional area DGPS system using multiple days of data. Proceedings of the 11th international technical meeting of the satellite division of the U.S. Institute of Navigation, 15-18 September 1998, Nashville, Texas, USA, 399-406.

Landau, H., Vollath, U., Deking, A. and Pagels, C. (2001). Virtual Reference Station Networks - Recent Innovations by Trimble. Proceedings of GPS symposium 2001, 14-16 November 2001, Tokyo, Japan.

Landau, H., Vollath, U. and Chen, X. (2002). Virtual Reference Station Systems. Journal of Global Positioning Systems, 1(2), 137-143.

Landau, H., Vollath, U. and Chen, X. (2003). Virtual Reference Stations versus Broadcast Solutions in Network RTK -Advantages and Limitations. Proceedings of GNSS 2003, April 2003, Graz, Austria.

Melbourne, W. G. (1985). The case for ranging in GPS based geodetic systems. Proceedings of the First International Symposium on Precise Positioning with the Global Positioning System, 15-19 April 1985, Rockville, Maryland, USA, 373-386.

Mohino, E., Gende, M. and Brunini, C. (2007). Improving long baseline (100-300 km) differential GPS positioning applying ionospheric corrections derived from multiple reference stations. Journal of Surveying Engineering, 133(1), 1-5. doi: 10.1061/ (ASCE)0733-9453.

Peter, F. (1999). Mesh Generation for Technology CAD in Three Dimensions. Dissertation, Technische Universitat, Wien, Fak. für Elektrotechnik.

Rizos, C. (2002). Network RTK research and implementation: A geodetic perspective. Journal of Global Positioning Systems, 2, 144-150.

Sun, H., Cannon, M. E. and Miligard, T. (1999). Real-time GPS reference network carrier-phase ambiguity resolution. Proceedings of the Institute of Navigation NTM-99, Institute of Navigation, San Diego, Alexandria, Virginia.

Tang, W., Liu, J., Liu, H. and Bai, Q. (2007). A Modified Combined Bias Interpolation Method for GNSS Network RTK. Geomatics and Information Science of Wuhan University, 32(12), 1156-1159 (in Chinese).

Teunissen, P. J. G. (1994). A new method for fast carrier phase ambiguity estimation. Proceedings of the IEEE Position, Location and Navigation Symposium (PLANS) '94, 11-15 April 1994, Las Vegas, Nevada, 562-573.

Teunissen, P. J. G. (1995a). The least-squares ambiguity de-correlation adjustment: a method for fast GPS integer ambiguity estimation. Journal of Geodesy, 70(1-2), 65-82. 
Teunissen, P. J. G., de Jonge, P. J. and Tiberius, C. C. J. M. (1995b). The LAMBDA method for fast GPS surveying. Proceedings of International Symposium 'GPS technology applications', 26-29 September 1995, Bucharest, Romania, 203-210.

Vollath, U., Birnbach, S., Landau, H. et al., (1999). Analysis of Three-Carrier Ambiguity Resolution (TCAR) Technique for Precise Relative Positioning in GNSS-2. Journal of The Institute of Navigation, 46(1), 13-23.

Wielgosz, P., Grejner-Brzezinska, D. and Kashani, I. (2004). Network Approach to Precise GPS Navigation. Journal of the Institute of Navigation, 51(3), 213-220.

Wübbena, G. (1985). Software developments for geodetic positioning with GPS using TI 4100 code and carrier measurements. Proceedings of the First International Symposium on Precise Positioning with the Global Positioning System, 15-19 April 1985, Rockville, Maryland, USA, 403-412.

Wübbena, G., Bagge, A., Seeber, G., Boder, V. and Hankemeier, P. (1996). Reducing distance dependent errors for real-time precise DGPS applications by establishing reference station networks. Proceedings of the 9 th international technical meeting of the Satellite Division of the U.S. Institute of Navigation, Kansas City, USA, 1845-1852.

Wübbena, G. (1989). The GPS adjustment software package-GEONAP-concepts and models. Proceedings of the 5th International Geodetic Symposium on Satellite Positioning, 13-17 March 1989, Las Cruces, New Mexico, 52-461.

Zheng, Y. and Feng, Y. (2005). Interpolating Residual Zenith Tropospheric Delays for Improved Regional Area Differential GPS Positioning, Navigation. Journal of the Institute of Navigation, 52(3), 179-187.

Zheng, Y., Feng, Y. and Bai, Z. (2006). Grid residual tropospheric corrections for improved differential GPS positioning over the Victoria GPS Network (GPSnet). Journal of Global Positioning Systems, 4(1-2), 284-290. 